\title{
Integration Capacity of Human Induced Pluripotent Stem Cell-Derived Cartilage
}

\author{
Xike Chen, MD, Akihiro Yamashita, DDS, PhD, Miho Morioka, BS,, Toshika Senba, VT, \\ Takashi Kamatani, MD, Akira Watanabe, PhD, ${ }^{2}$ Azuma Kosai, DDS, and Noriyuki Tsumaki, MD, PhD ${ }^{1}$
}

New cell and tissue sources are needed for the regenerative treatment of articular cartilage damage. Human induced pluripotent stem cells (hiPSCs) are an abundant cell source due to their self-renewal capacity. Hyaline cartilage tissue particles derived from hiPSCs (hiPS-Carts), $1-3 \mathrm{~mm}$ in diameter, are one candidate source that can be used for transplantation. When transplanted to fill the defects of articular cartilage, hiPS-Carts form a repair tissue by integrating with each other and with adjacent host tissue. In this study, we analyzed the integration capacity using an in vitro model and found that hiPS-Carts spontaneously integrate with each other in vitro. hiPS-Carts consist of cartilage at the center and perichondrium-like membrane that wraps around the cartilage. The integration started at the perichondrium-like membrane at around 1 week. Then, the integration progressed to the cartilage within 4-8 weeks. RNA sequencing analysis identified a higher expression of FGF18 in the perichondrium-like membrane in hiPS-Carts compared with the central cartilage. The addition of FGF18 to the model accelerated the integration of hiPS-Carts, whereas the addition of a FGFR inhibitor inhibited it. These results suggest that FGF18 secreted from the perichondrium-like membrane plays a role in the integration of hiPS-Carts. Understanding the integration mechanism of hiPS-Carts is expected to contribute to the realization of regenerative treatment for patients with articular cartilage damage.

Keywords: cartilage, iPS cells, perichondrium, FGF, chondrocyte

\section{Impact Statement}

Cartilage particles derived from human induced pluripotent stem cells (hiPS-Carts) are one candidate source for transplants for treatment of articular cartilage damage. This study shows that hiPS-Carts integrate with each other in an in vitro model and analyzed the course of the integration. The integration starts at the perichondrium-like membrane at around 1 week and then progresses to the central cartilage within 4-8 weeks. The results indicate that FGF18 secreted from the perichondriumlike membrane accelerates the initial step of integration. The findings contribute to understanding how hiPS-Carts form repair tissue and provide clue to accelerate healing after transplantation.

\section{Introduction}

A RTICULAR CARTILAGE COVERS the ends of bones and provides lubrication between opposing bones during joint motion. It consists of chondrocytes and the cartilage extracellular matrix (ECM), which embeds the chondrocytes. Chondrocytes produce cartilage ECM, while cartilage ECM provides chondrocytes with an environment that supports the chondrocytes to sustain chondrocytic characters, including the production of cartilage ECM, indicating a mutually dependent relationship.
Damage to cartilage seldom heals because it causes a loss of cartilage ECM. Cells cannot be chondrocytes in the absence of cartilage ECM, ${ }^{1-3}$ and nonchondrocytic cells do not produce cartilage ECM in the defect in the joint surface created by the damage. Although recent regenerative treatments have employed the implantation of autologous chondrocytes or mesenchymal stem cells into articular cartilage defects, these cell implantations without cartilage ECM do not reproduce cartilage, as indicated by the formation of fibrocartilaginous repair tissue. $^{4-7}$ To overcome this limitation, the implantation of

\footnotetext{
${ }^{1}$ Cell Induction and Regulation Field, Department of Clinical Application, Center for iPS Cell Research and Application, Kyoto University, Kyoto, Japan.

${ }^{2}$ Department of Life Science Frontiers, Center for iPS Cell Research and Application, Kyoto University, Kyoto, Japan.

(C) Xike Chen et al. 2018; Published by Mary Ann Liebert, Inc. This Open Access article is distributed under the terms of the Creative Commons Attribution Noncommercial License (http://creativecommons.org/licenses/by-nc/4.0/) which permits any noncommercial use, distribution, and reproduction in any medium, provided the original author(s) and the source are cited.
} 
allogeneic cartilage has been employed. Because cartilage has low immunogenicity, ${ }^{8,9}$ articular cartilage damage has been treated by transplanting allogeneic cartilage without matching HLA types or use of immunosuppressive drugs. ${ }^{9-13}$ While promising results have been obtained, the lack of donors, heterogeneous quality of the cartilage obtained from different individuals, and risk of disease transmission suggest an alternative method is preferred. In particular, new materials that can be transplanted into articular cartilage defects as regenerative treatment for joint injury are desired. ${ }^{14}$

Induced pluripotent stem cells (iPSCs) ${ }^{15,16}$ are a potential cell source for all cell types, including chondrocytes. iPSCs are somatic cells, such as skin cells or blood cells, that have been reprogrammed to the pluripotent state by the introduction of specific factors. iPSCs have similar properties, such as pluripotency and self-renewal, with embryonic stem cells, but do not require the sacrifice of embryos. We previously reported a method to generate scaffoldless cartilage tissues from human iPSCs (hiPSCs). ${ }^{17,18}$ Three-dimensional culture of chondrogenically-differentiated hiPSCs in suspension culture causes these cells to secrete and deposit cartilage ECM to form cartilage. These hiPSC-derived cartilages (hiPS-Carts) are spherical and about $1-3 \mathrm{~mm}$ in diameter. When hiPS-Carts were transplanted into the defective articular cartilage of minipigs that were treated with immunosuppressive drugs, they constituted articular cartilage and showed indications of integration with native mini-pig articular cartilage at 4 weeks after transplantation. ${ }^{17}$ When hiPS-Carts were transplanted into the defective articular cartilage of immunodeficient rats, they integrated with adjacent rat native cartilage at the sides and bone at the bottom at 4 weeks after transplantation. ${ }^{17}$ In addition, we found that hiPS-Carts have low immunogenicity similar to natural cartilage, as indicated by the lack of proliferation of lymphocytes in a mixed lymphocyte reaction assay. ${ }^{19}$ These findings collectively suggest that hiPS-Carts can be a new source for allogeneic transplantation to treat defects of articular cartilage. The numbers of hiPS-Carts needed to fill a defect depend on the size of the defect in the articular cartilage. In a clinical setting, we are planning to implant around 25 hiPS-Cart per $1 \mathrm{~cm}^{2}$ defect and fix them with fibrin glue. To heal the defect, hiPS-Carts must integrate with each other and the host cartilage; however, the integrating process of hiPSCarts is not known.

In the present study, we report that hiPS-Carts have the capability to integrate with each other when left attached in vitro. Using this in vitro model, we analyzed the mechanisms of hiPS-Cart integration and investigated methods that promote the integration.

\section{Materials and Methods}

\section{Ethics statement}

All experiments were approved by the institutional review board, institutional animal committee (as appropriate), and the institutional biosafety committee of Kyoto University.

\section{Cells and tissues}

hiPSCs: Four iPSC lines, 409B2, 604B1, 1231A3 and QHJ, were generated from different healthy individuals and were gifted by K. Okita, M. Nakagawa, and S. Yamanaka (Center for iPS Cell Research and Application [CiRA], Kyoto Uni- versity, Kyoto, Japan). ${ }^{20}$ 409B2 was generated from human dermal fibroblasts, and 604B1, 1231A3, and QHJI from human peripheral mononuclear cells. Episomal plasmid vectors (pCXLE-hOCT3/

4-shp53-F, hSK, hUL, EBNA1) were electroporated, and all cells were negative for genome integration.

hiPS-Carts: hiPSCs were chondrogenically differentiated to produce hiPS-Carts following a previously described method $^{17}$ with modification. hiPS-Carts generated by chondrogenic differentiation for 12 weeks were used in the experiments.

\section{Integration of hiPS-Carts in vitro}

Two hiPS-Carts were put into a well of a round-bottom 96well plate (Fig. 1A) and cultured in chondrogenic medium (DMEM [Sigma] with 1\% ITS, 1\% FBS, 2 mM L-glutamine [Thermo], $1 \times 10^{-4} \mathrm{M}$ nonessential amino acids [Thermo], $1 \mathrm{mM}$ sodium pyruvate [Thermo], $50 \mathrm{U}$ penicillin, $50 \mathrm{mg} / \mathrm{mL}$ streptomycin, $50 \mu \mathrm{g} / \mathrm{mL}$ ascorbic acid [Nacalai], $10 \mathrm{ng} / \mathrm{mL}$ BMP2 [Peprotech], $10 \mathrm{ng} / \mathrm{mL}$ TGF $\beta 1$ [Peprotech] and $10 \mathrm{ng} /$ mL GDF5 [PTT]). ${ }^{17}$ In total, 84 wells, each of which contained a pair of hiPS-Carts, were prepared. Wells were divided into seven groups (12 pairs per group), and each was subjected to macroscopic and histological analyses at a respective time point $(0,3,7,10,14,28$, or 56 days after the start of the integration experiment). We mildly changed the medium every day, paying attention not to change the positions of hiPS-Carts.

\section{Time-lapse imaging of integration}

hiPS-Carts were prepared from 201B7 hiPSC lines bearing either CAG-EGFP (317-12) or CAG-mCherry (511-5B) transgenes targeted to the AAVS1 locus. ${ }^{21}$ The integration of an EGFP hiPS-Cart and a mCherry hiPS-Cart was subjected to time-lapse observation using a multiphoton laser microscope (Nikon A1R MP+) and analysis software (Nikon NIS Elements). Fluorescent images were captured every $1 \mathrm{~h}$ for 11.5 consecutive days. Each image in the movie represents $100 \mathrm{~ms}$; thus $24 \mathrm{~h}$ corresponds to $2.4 \mathrm{~s}$. The time-lapse images were interrupted several times when the iPS-Carts moved and went out of the field of view.

\section{Manipulation of FGF signaling during integration of hiPS-Carts}

Recombinant human FGF18 (PeproTech) was dissolved in phosphate-buffered saline (PBS) to prepare a stock solution $(100 \mu \mathrm{g} / \mathrm{mL})$. In total, 90 pairs of hiPS-Carts were cultured in the condition described above. One pair per well was cultured in $0.3 \mathrm{~mL}$ medium. Forty-five pairs were cultured in the medium supplemented with vehicle $(0.3 \mu \mathrm{L}$ PBS $)$, and 45 pairs were cultured in the medium supplemented with $0.3 \mu \mathrm{L}$ FGF18 stock solution (final concentration $100 \mathrm{ng} / \mathrm{mL}$ ). The 45 pairs in each treatment group were further separated into three equally-sized groups and subjected to histological analysis $(3,7$, or 14 days after the start of the experiment).

To further investigate the effects of FGF on the integration, a FGF inhibitor, NVP-BGJ398 (ChemScene LLC), was used in the culture. NVP-BGJ398 was dissolved in DMSO to prepare $50 \mu \mathrm{M}$ stock solution. In total, 30 new pairs of hiPS-Carts were cultured in the condition described above. 
A
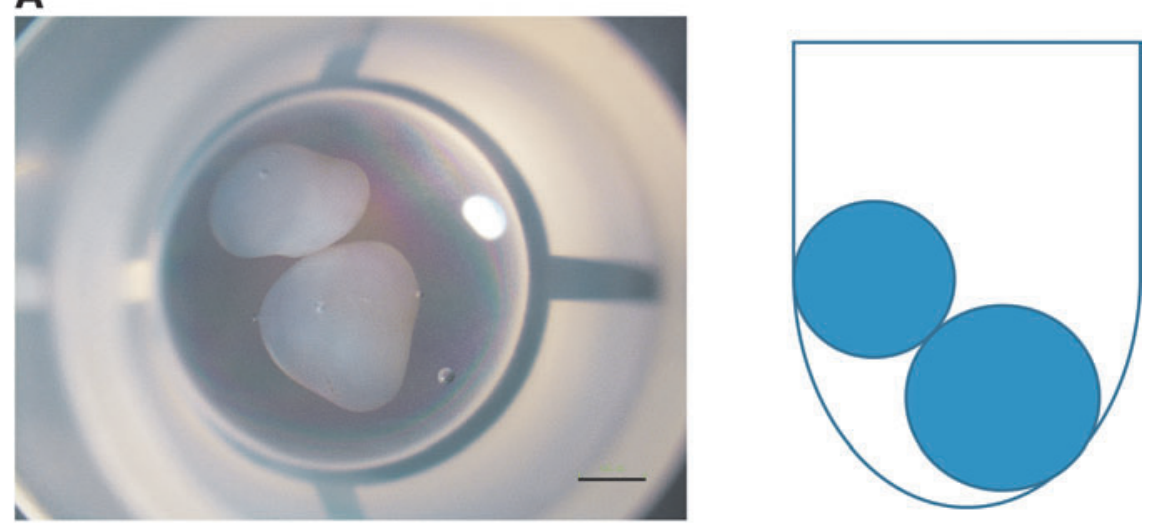

B

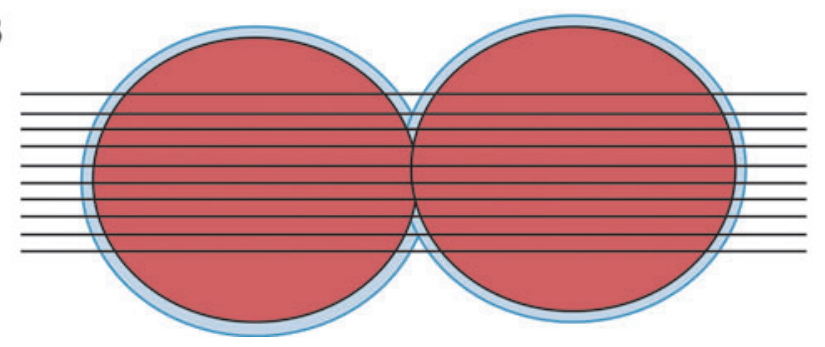

Fifteen pairs were cultured in medium supplemented with vehicle $(0.3 \mu \mathrm{L}$ DMSO), and the other 15 pairs were cultured in medium supplemented with $0.3 \mu \mathrm{L}$ NVP-BGJ398 stock solution (final concentration $50 \mathrm{nM}$ ). After 14 days of culture, the samples were subjected to histological analysis.

\section{Histological analysis}

Pairs of hiPS-Carts were fixed with $4 \%$ paraformaldehyde, processed, and embedded in paraffin. To secure the sections that covered the most contacting area between the pairs, we prepared 20-150 serial sections around the integrated portion of the sample (Fig. 1B). Serial sections with the greatest contacting portion were selected and used for further analysis. The sections were stained with hematoxylin-eosin and safranin Ofast green-iron hematoxylin and immunostained with goat antitype I collagen antibody (Souther Biotech) and anti-type II collagen antibody (Thermo), as described previously. ${ }^{17}$

\section{RNA extractions from perichondrium-like membrane and central cartilage}

We peeled QHJI hiPS-Carts to separate the perichondriumlike membrane from the central cartilage using forceps under stereomicroscopy. RNAs were extracted separately from the perichondrium-like membrane and central cartilage. Samples were frozen in liquid nitrogen and crushed using Multi Beads Shocker (Yasui Kikai, Osaka, Japan), and total RNA was extracted using ISOGEN ${ }^{\circledR}$ (Nippon Gene) and purified with RNeasy (Qiagen).

\section{RNA sequencing analysis}

The quality of the extracted RNAs was evaluated using Bioanalyzer 2100 (Agilent Technologies). One microgram of total RNA was subjected to library preparation using TruSeq Stranded mRNA Library Prep Kit (Illumina) according to the manufacturer's instruction. The quality and
Serial

sections
FIG. 1. Experimental methods for integration of hiPS-Carts. (A) Left: An image of a well of a round-bottom 96-well plate that contains a pair of hiPS-Carts. Scale bar, $1 \mathrm{~mm}$. Right: Schematic image of the well. Blue circles indicate hiPS-Carts. (B) Schematic explanation of the sites of the histological serial sections. About 20-150 serial sections around the integrating portion of the sample were prepared. Sections that covered most of the contacting portion were selected and used for further assessment. quantity of the constructed libraries were evaluated using Bioanalyzer 2100 and Qubit dsDNA HS assay kit (Thermo). The libraries were sequenced in 75-cycle single-read mode of NextSeq 500 (Illumina). All sequence reads were extracted in FASTQ format using BCL2FASTQ Conversion Software (v2.17.1.14.). The adaptors, the poly-A sequences, and the low-quality bases at the $3^{\prime}$ read ends were trimmed using cutadapt-1.12. Untrimmed and trimmed reads were mapped onto the human genome hg38 using TopHat-2.1.1. We utilized human gene annotation from GENCODE release v25. For gene expression analysis, the expression level of each gene was normalized to reads per kilobase of exon per million sequence reads (RPKM) using Cufflinks-2.2.1.

\section{Real-time reverse transcription-polymerase chain reaction expression analysis}

We extracted total RNAs separately from three perichondrium-like membrane samples, three central cartilage samples, and three whole hiPS-Carts. Five hundred nanograms of total RNA was reverse-transcribed into first-strand cDNA using ReverTra Ace (Toyobo, Tokyo, Japan) and an oligo(dT) 20 primer. PCR amplification was performed using KAPA PROBE FAST qPCR kit or KAPA SYBR FAST qPCR kit Master Mix ABI prism (KAPA Biosystems, MA). The PCR primers used are listed in Table 1. The RNA

Table 1. Primers Used for Real-Time Reverse Transcription-Polymerase Chain Reaction

\begin{tabular}{ll}
\hline Primer & \multicolumn{1}{c}{ Sequence or TaqMan ID } \\
\hline FGF18 forward & CACTTTCTACTGCTGCTGCTTCCA \\
FGF18 reverse & GCATACTTGTCCCCGTCCTC \\
COL1A1 & Hs00164004_m1 \\
COL2A1 & Hs00264051_m1 \\
SOX9 & Hs01001343_g1 \\
GAPDH & Hs03929097_g1 \\
\hline
\end{tabular}


FIG. 2. Course of integration between hiPS-Carts in vitro. $(\mathbf{A}, \mathbf{B}) \mathrm{A}$ pair of QHJI hiPS-Carts was left attached passively in the culture. (A) Samples were analyzed after 3 , 7, 10, 14, 28, 56, and 84 days. Left column: Macroscopic images of a pair of hiPS-Carts. Second column: Histological section stained with safranin $\mathrm{O}$-fast green-iron hematoxylin. Third column: Magnification of the boxed region in the second column. Right column: Magnification of the boxed region in the third column (days 14 and 28) or second column (days 56 and 84). Scale bar, $500 \mu \mathrm{m}$. (B) Samples were analyzed after 7, 14, and 84 days. Histological sections of integrating hiPS-Carts were immunostained with antitype I collagen antibody. Boxed regions in the left column are magnified in the second and third columns. The semiserial section in the third column on day 84 was immunostained with anti-type II collagen antibodies. Scale bar, $500 \mu \mathrm{m}$. (C) Histology of integrating hiPS-Carts created from independent hiPSC lines (409B2, 604B1, and 1231A3). Scale bar, $500 \mu \mathrm{m}$. hiPSC, human induced pluripotent stem cell.
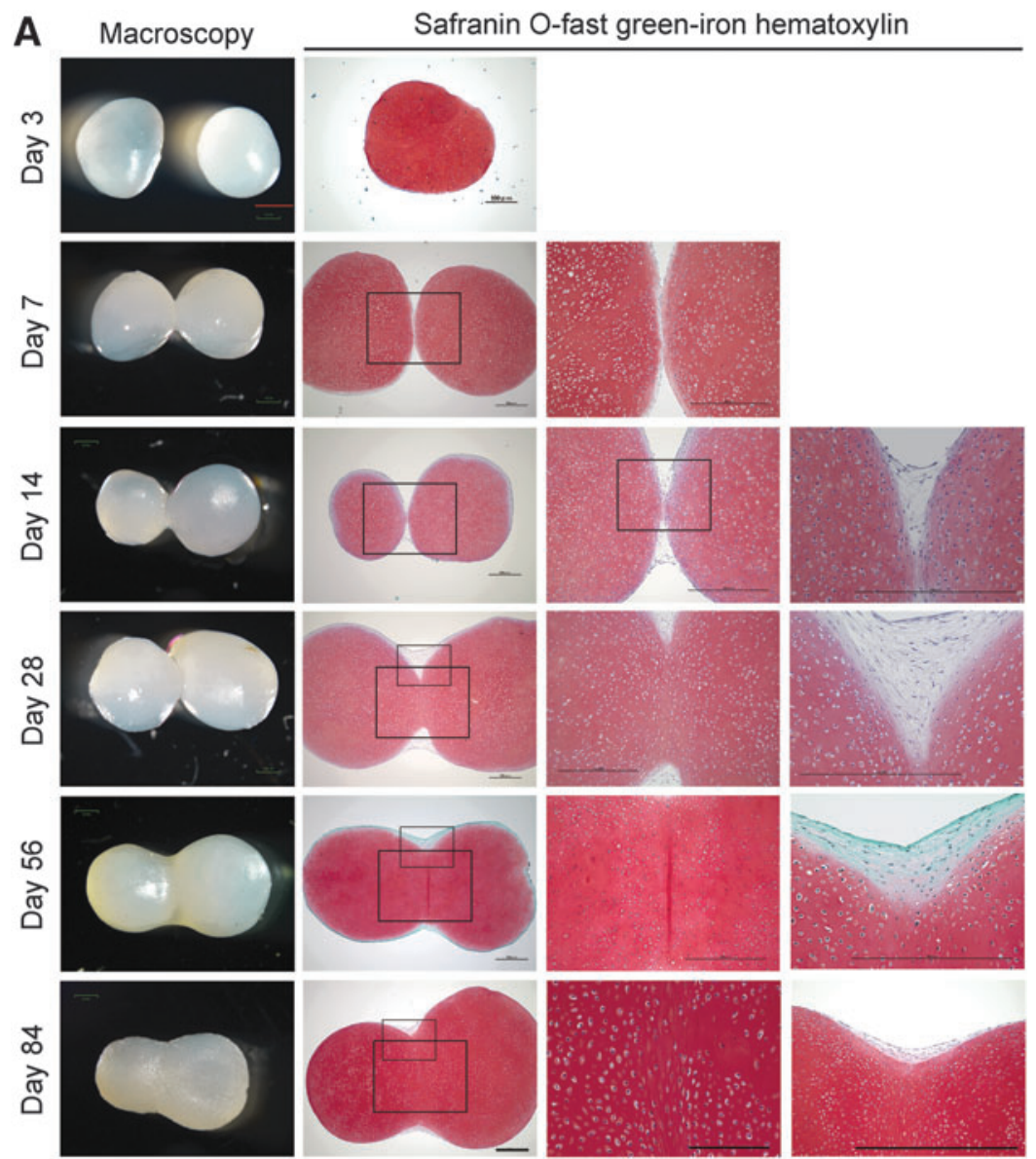

B

COL1

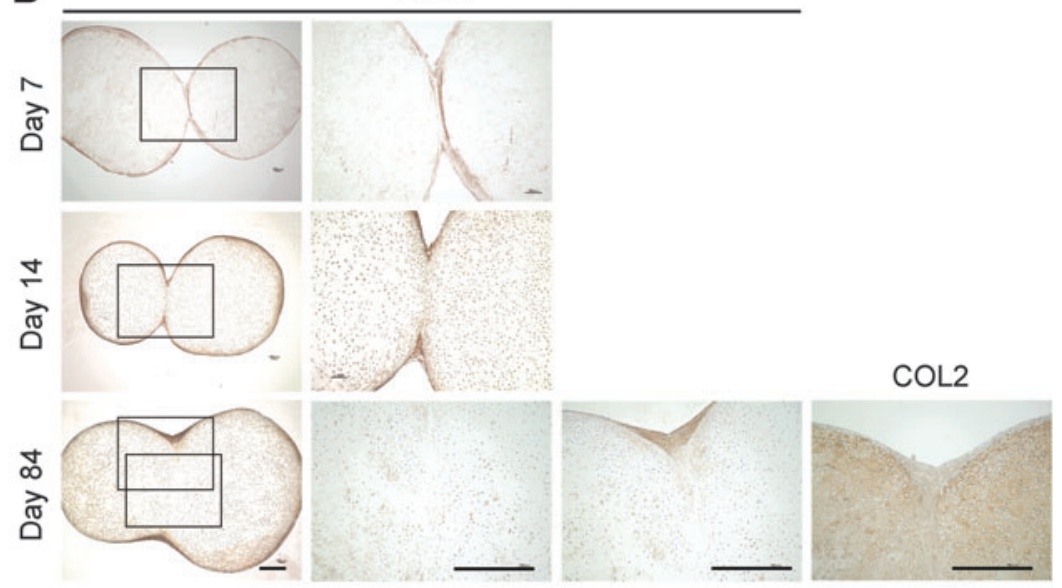

C

409B2

604B1

$1231 \mathrm{A3}$
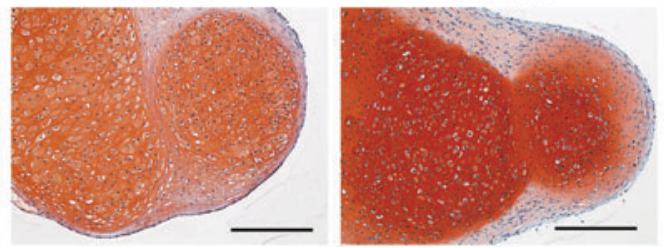
expression levels were normalized to the level of GAPDH expression. Amplified products were used to derive standard curves for real-time quantitative reverse transcriptionpolymerase chain reaction (RT-PCR).

\section{Statistical analysis}

The data are shown as averages and standard deviations. In this study, we used the two-tailed Student's $t$-test or Steel-Dwass test. $p$-Values $<0.05$ were considered to be statistically significant.

\section{Results}

Analysis of the course of integration between QHJI hiPS-Carts in vitro

Two hiPS-Carts started to bond after 7 days and proceeded to form a solid union after that (Fig. 2A, macroscopic image). Histologically, one hiPS-Cart consists of cartilage at the center and perichondrium-like membranous tissue that wraps around the cartilage (Fig. 2A, safranin O staining). On day 7, when the hiPS-Carts started integrating, only the perichondrium-like membranes were integrated, whereas the cartilages of the hiPS-Carts were separated by the membrane. A portion of the perichondrium-like membrane at the integrated site was thickened on days 14, 28, and 56 (Fig. 2A, right column), suggesting that cells in the perichondrium-like membrane had proliferated. On day 14, we found portions of the cartilage of the two hiPS-Carts were in contact. The contact became substantial on day 28 , and the cartilage had integrated by days 56 and 84 (Fig. 2A, second and third columns).

The perichondrium-like membrane in hiPS-Carts expressed type I collagen (Fig. 2B). In contrast, central cartilage expressed type II collagen. On day 7, we detected a clear line (Fig. 2B, square), indicating type I collagen expression in the contact face between the two hiPS-Carts and that the contact was between the perichondrium-like membranes. On day 14, the type I collagen-expressing line was disappearing, and by day 84 it had completely disappeared, indicating that the two hiPS-Carts were integrated by cartilage. Type II collagen was expressed in the connected site as strongly as it was in the central cartilage (Fig. 2B, right column).

\section{Statistical analysis of integration progression of hiPS-Carts}

For statistical analysis, we graded the degree of integration of hiPS-Carts at each time point: grade 0, no integration; grade 1, integration by perichondrium-like membrane; grade 2 , integration by cartilage (Table 2). Because the grades are nonparametric data, we performed statistical analysis with the Steel-Dwass test. All samples were grade 0 (no integration) on day 0 and grade 2 (integration by cartilage) on day 56. The grades on the time points after day 7 were significantly different from that on day 0 (Table 3 ). The grades on the time points before day 14 were significantly different from those on day 56.

\section{Integration of hiPS-Carts was reproduced in different hiPSC lines}

To confirm that our findings applied to multiple iPSC lines, we created hiPS-Carts from 409B2, 604B1, and 1231A3 as
Table 2. Number of QHJI Human Induced

Pluripotent Stem-Cart Pairs by Grade OF INTEGRATION

\begin{tabular}{|c|c|c|c|c|}
\hline & \multirow{2}{*}{ 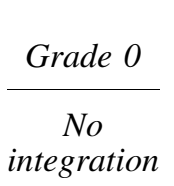 } & \multirow{2}{*}{$\begin{array}{l}\text { Grade } 1 \\
\text { Integration by } \\
\text { perichondrium- } \\
\text { like membrane }\end{array}$} & \multirow{2}{*}{ 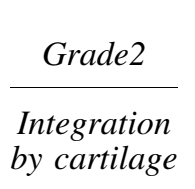 } & \multirow[b]{2}{*}{ Total } \\
\hline & & & & \\
\hline Day 0 & 12 & 0 & 0 & 12 \\
\hline Day 3 & 12 & 0 & 0 & 12 \\
\hline Day 7 & 5 & 7 & 0 & 12 \\
\hline Day 10 & 2 & 9 & 1 & 12 \\
\hline Day 14 & 0 & 8 & 4 & 12 \\
\hline Day 28 & 0 & 5 & 7 & 12 \\
\hline Day 56 & 0 & 0 & 12 & 12 \\
\hline
\end{tabular}

well. As with QHJI, when two hiPS-Carts were left attached in the culture dish, they spontaneously integrated gradually, and histological analysis confirmed the integration (Fig. 2C).

\section{Time-lapse imaging of hiPS-Carts integration}

Time-lapse imaging of the integration indicated that cells in the perichondrium-like membrane migrated into the perichondrium-like membrane of the other hiPS-Cart (Supplementary Video S1). This observation suggests that the perichondrium-like membrane plays a substantial role during integration.

\section{mRNA expression analysis of perichondrium-like membrane of hiPS-Cart}

Histological analysis of dissected samples confirmed separation between the perichondrium-like membrane and central cartilage (Fig. 3A). RNAs were extracted from the perichondrium-like membrane and the central cartilage separately and subjected to RNA sequencing analysis. The RPKM values for $C O L 2 A 1$ and $S O X 9$ were higher in central cartilage than in perichondrium-like membrane, whereas the RPKM values for COL1A1 were vice versa (Fig. 3B), suggesting that the sampling and RNA sequencing experiments were performed well. Among differentially expressed genes, we found FGF18 mRNA was expressed more in the perichondrium-like membrane than in the central cartilage (Fig. 3B, C and Table 4). We confirmed the differential

Table 3. Statistical Analysis of Table 2

\begin{tabular}{lccccccc}
\hline & $\begin{array}{c}\text { Day } \\
0\end{array}$ & $\begin{array}{c}\text { Day } \\
3\end{array}$ & $\begin{array}{c}\text { Day } \\
7\end{array}$ & $\begin{array}{c}\text { Day } \\
10\end{array}$ & $\begin{array}{c}\text { Day } \\
14\end{array}$ & $\begin{array}{c}\text { Day } \\
28\end{array}$ & $\begin{array}{c}\text { Day } \\
56\end{array}$ \\
\hline Day 0 & & & & & & \\
Day 3 & N.S. & & & & & \\
Day 7 & a & a & & & & \\
Day 10 & b & b & N.S. & & & \\
Day 14 & b & b & a & N.S. & & \\
Day 28 & b & b & b & N.S. & N.S. & & b.S. \\
Day 56 & b & b & b & b & & N.S. \\
\hline
\end{tabular}

${ }^{\mathrm{a}} P<0.05$ and ${ }^{\mathrm{b}} P<0.01$ by Steel-Dwass test. N.S., not significant. 


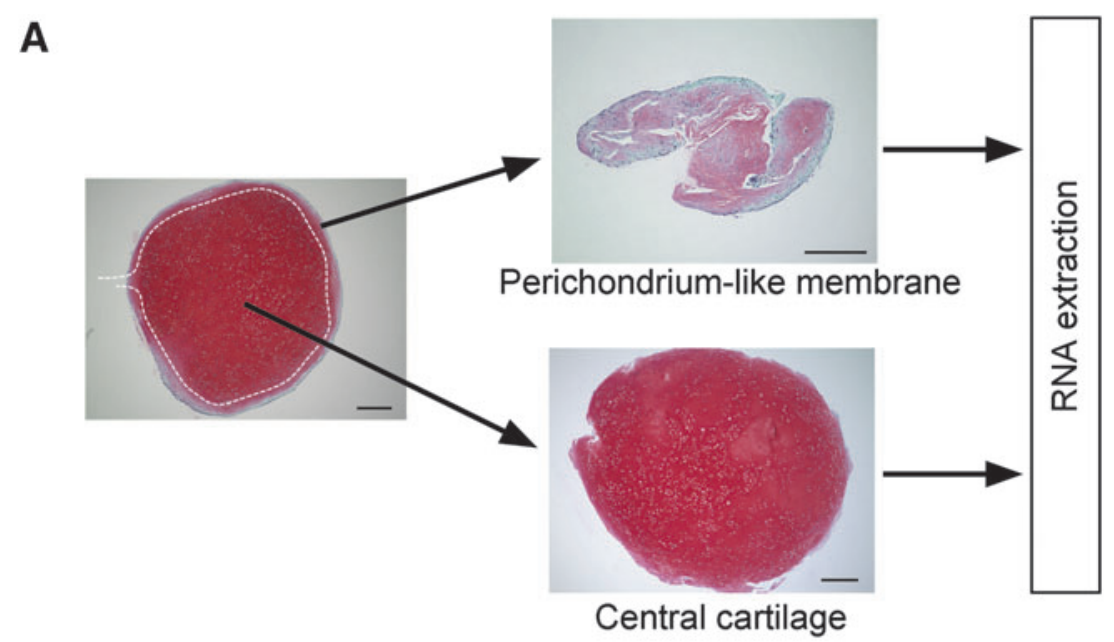

FIG. 3. mRNA expression analysis of perichondrium-like membrane of hiPSCart. (A) Preparation of the samples for RNA extraction. We peeled the hiPS-Cart to separate the perichondrium-like membrane from the central cartilage. Histological analysis of the samples was performed to confirm that the separation was done appropriately. Safranin O-fast green-iron hematoxylin staining. Scale bar, $500 \mu \mathrm{m}$. (B, C) Results of RNA sequencing transcriptome analysis of the perichondrium-like membrane and central cartilage. RPKM, reads per kilobase of exon per million sequence reads. Representative data from two independent experiments are shown. (B) Comparison of the RPKM for marker genes in the perichondrium-like membrane to those in the central cartilage. (C) Plots showing differences in gene expressions in the perichondrium-like membrane versus the central cartilage. Each dot represents one gene. The $y$-axis shows the fold change in the RPKM in perichondrium-like membrane versus that in central cartilage. The $x$-axis shows the $\log _{10}$ of the RPKM in the perichondrium-like membrane. The red arrow indicates the FGF18 gene. (D) Real-time RT-PCR expression analysis of marker genes in the perichondrium-like membrane and central cartilage. Relative expression levels in the perichondriumlike membrane are set as 1 . Error bars denote means \pm s.d. $* * p<0.01$ compared with central cartilage by Student's $t$-test ( $n=3$ technical replicates). Re-

presentative data from three independent experiments are shown. C, central cartilage of hiPS-Cart; $\mathrm{M}$, perichondrium-like membrane of hiPS-Cart; RT-PCR, reverse transcription-polymerase chain reaction.

\begin{tabular}{cccc}
\hline \multirow{2}{*}{ GENE } & \multicolumn{3}{c}{ RPKM } \\
\cline { 2 - 4 } & $\begin{array}{c}\text { Perichondrium-like } \\
\text { Membrane }(\mathrm{M})\end{array}$ & $\begin{array}{c}\text { Central } \\
\text { Cartilage }(\mathrm{C})\end{array}$ & $\mathrm{M} / \mathrm{C}$ \\
\hline SOX9 & 4.75 & 39.27 & 0.12 \\
COL2A1 & 200.95 & 6223.85 & 0.03 \\
COL1A1 & 628.33 & 282.44 & 2.23 \\
FGF18 & 3.48 & 0.42 & 8.29 \\
\hline
\end{tabular}

C

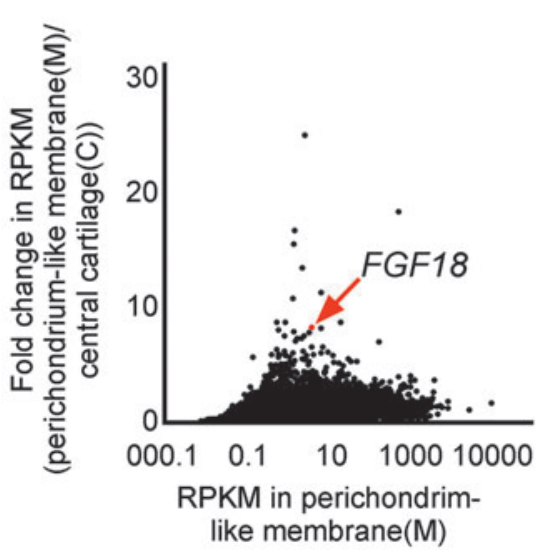

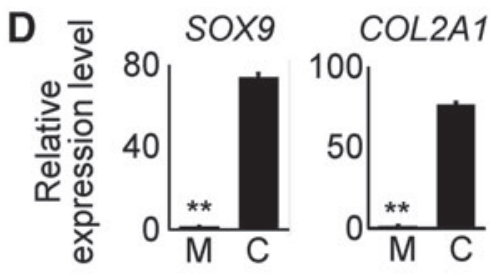

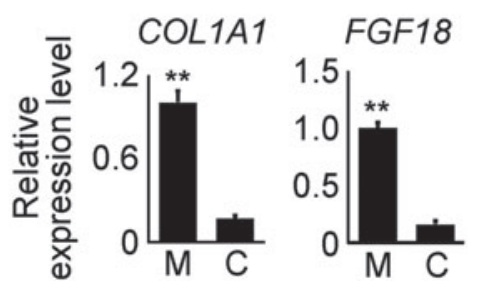

expression of these genes between the perichondrium-like membrane and central cartilage by real-time RT-PCR expression analysis (Fig. 3D).

\section{FGF18 expressed in the perichondrium-like membrane regulates integration of hiPS-Carts}

The addition of FGF18 to the medium accelerated the integration of hiPS-Carts. On day 14, vehicle-treated hiPSCarts showed minimal integration, whereas FGF18-treated hiPS-Carts showed substantial integration (Fig. 4). Histologically, FGF18-treated hiPS-Carts showed thick connections by the perichondrium-like membrane. We found a significant difference in the grades of integration between vehicle-treated and FGF18-treated pairs of hiPS-Carts on day 14 (Table 5).
In contrast, the addition of a FGFR inhibitor, NVPBGJ398, to the medium inhibited the integration of hiPSCarts. On day 14, 11 out of 15 pairs of vehicle-treated hiPS-Carts showed integration, whereas no pairs of NVPBGJ398-treated hiPS-Carts did (Fig. 5 and Table 6). We found a significant difference in the grades of integration between vehicle-treated and NVP-BGJ398-treated pairs of hiPS-Carts (Table 6).

\section{Discussion}

Here we show that hiPS-Carts initially integrate with each other at the perichondrium-like membrane and then subsequently at the central cartilage in vitro. The integration of hiPS-Carts in vitro is a common event of hiPSC-derived cartilage, because the integration was reproducible in hiPS- 
Table 4. Most Differentially Expressed Genes Between Perichondrium-Like Membrane and Central Cartilage

\begin{tabular}{lccc}
\hline \multirow{4}{*}{ Gene } & \multicolumn{3}{c}{ RPKM } \\
\cline { 2 - 4 } & $\begin{array}{c}\text { Perichondrium-like, } \\
\text { Membrane }(M)\end{array}$ & $\begin{array}{c}\text { Central, } \\
\text { Cartilage }(C)\end{array}$ & M/C \\
\hline CHST9 & 2.24 & 0.09 & 25.07 \\
CHI3L1 & 397.46 & 21.62 & 18.38 \\
POLM & 1.27 & 0.08 & 16.76 \\
GGT7 & 1.17 & 0.08 & 15.58 \\
WISP2 & 1.90 & 0.14 & 13.46 \\
NRN1 & 5.45 & 0.48 & 11.29 \\
SLC22A1 & 1.12 & 0.10 & 10.79 \\
ZNF354C & 0.73 & 0.08 & 8.74 \\
C2orf91 & 0.47 & 0.05 & 8.74 \\
ADAMTSL1 & 15.90 & 1.82 & 8.72 \\
FGF18 & 3.48 & 0.42 & 8.19 \\
CD70 & 5.34 & 0.65 & 8.18 \\
NELL1 & 0.51 & 0.06 & 8.03 \\
CD74 & 1.20 & 0.15 & 7.86 \\
C3 & 2.75 & 0.35 & 7.84 \\
\hline
\end{tabular}

RPKM, reads per kilobase of exon per million sequence reads.

Carts from different hiPSC lines. RNA sequencing analysis revealed that the perichondrium-like membrane expressed higher FGF18 than the central cartilage. Addition of FGF18 accelerated the integration and increased the thickness of the perichondrium-like membrane. These results, together with our time-lapse imaging of migration of cells in perichondrium-like membrane during the integration, suggest that FGF18 accelerates the integration by stimulating cells in the perichondrium-like membrane. The result that the addition of FGFR inhibitor inhibited integration further supports the speculation that FGF18 secreted from perichondrium contributes to integration. Regarding the source of FGFs, we cannot exclude the possible contribution of FGFs in FBS in the medium, but we speculate that effect was weak because the concentration of FBS we used was low (1\%). Because a previous report found that FGF18 is highly expressed in perichondrium ${ }^{22}$ and because we found FGF18 was specifically expressed in the perichondrium-like membrane in hiPS-Carts, the perichondrium-like membrane of hiPS-Carts may correspond to perichondrium.

There are two possible courses that explain how the membranous integration became cartilaginous integration. One is that the membrane at the contacting area is resorbed, and subsequently the cartilage of both hiPS-Carts expand and integrate with each other. The other is that cells in the membrane turn into chondrocytes, which then produce cartilage that integrate together at the center of the two hiPSCarts. Further experiments are required to identify which of the two mechanisms accurately describes the integration process.

Our result that FGF18 accelerates integration in vitro suggests that the healing process after the transplantation of hiPS-Carts into articular cartilage defects will be enhanced by administering FGF18. However, we should note the differential effects of FGF signaling on different types of cells. ${ }^{23}$ FGF signaling stimulates the proliferation of most cell types, including fibroblasts, but it inhibits the

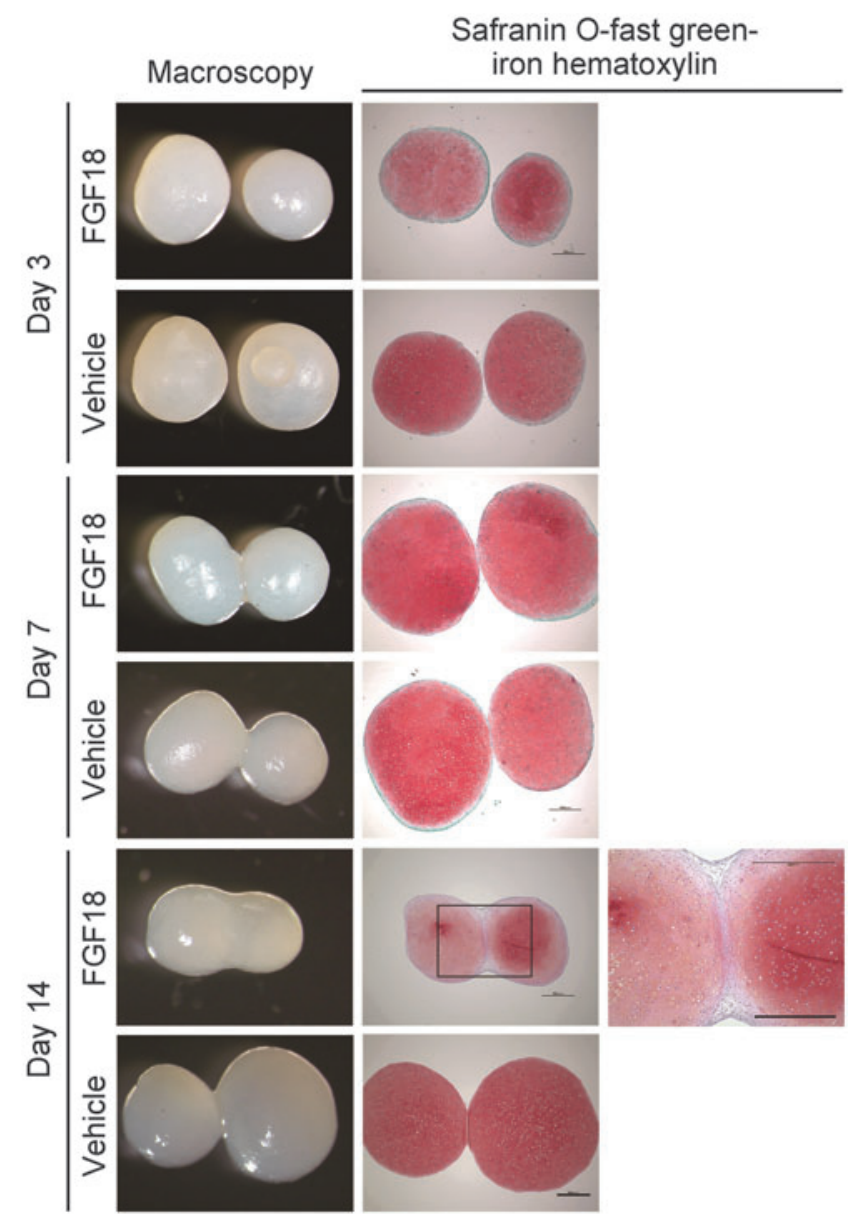

FIG. 4. FGF18 regulates integration of hiPS-Carts. Pairs of QHJI hiPS-Carts were cultured in the presence of vehicle or $100 \mathrm{ng} / \mathrm{mL}$ FGF18 for the periods indicated. Left column: Macroscopic images of a pair of hiPS-Carts. Central column: Histological section stained with safranin O-fast green-iron hematoxylin. Right column: Magnification of the boxed region in the central column. Scale bar, $500 \mu \mathrm{m}$.

Table 5. Number of QHJI Human Induced Pluripotent Stem-Cart Pairs by Grade OF INTEGRATION IN RESPONSE TO FGF18

\begin{tabular}{|c|c|c|c|c|}
\hline & \multirow{2}{*}{ 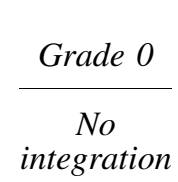 } & \multirow{2}{*}{ 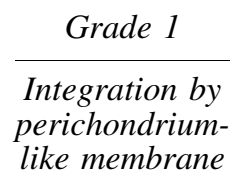 } & \multirow{2}{*}{ 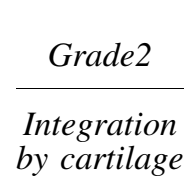 } & \multirow{2}{*}{ Total } \\
\hline & & & & \\
\hline \multicolumn{5}{|l|}{ Day 3} \\
\hline Vehicle & 14 & 1 & 0 & 15 \\
\hline FGF18 & 12 & 3 & 0 & 15 \\
\hline \multicolumn{5}{|l|}{ Day 7} \\
\hline Vehicle & 12 & 3 & 0 & 15 \\
\hline FGF18 & 10 & 5 & 0 & 15 \\
\hline \multicolumn{5}{|l|}{ Day 14} \\
\hline Vehicle & 9 & 5 & 1 & 15 \\
\hline FGF18 & 1 & 14 & 0 & 15 \\
\hline
\end{tabular}

${ }^{\mathrm{a}} P<0.01$ compared with vehicle by the Steel-Dwass test. 


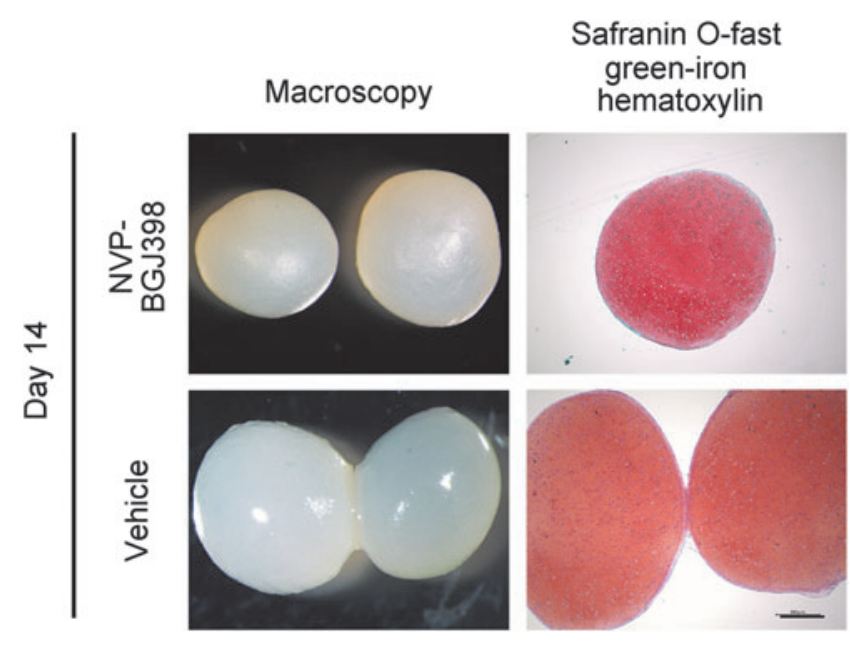

FIG. 5. FGFR inhibitor inhibits integration of hiPS-Carts. Pairs of QHJI hiPS-Carts were cultured in the presence of vehicle or $10 \mathrm{nM}$ NVP-BGJ398 for 14 days. Left column: Macroscopic images of a pair of hiPS-Carts. Right column: Histological section stained with safranin O-fast green-iron hematoxylin. Scale bar, $500 \mu \mathrm{m}$.

proliferation and differentiation of chondrocytes. ${ }^{24}$ FGF18 has been reported to affect proliferation, differentiation, and ECM production in a context-dependent manner. ${ }^{25,26}$ In the present study, although the connected area was thick in FGF18-treated hiPS-Cart pairs, all pairs were connected only by the perichondrium-like membrane and not by the cartilage on day 14 (Fig. 4 and Table 5). In addition, safranin $\mathrm{O}$ staining intensities in the cartilaginous portion of hiPS-Carts were reduced in FGF18-treated hiPS-Cart pairs on day 14 (Fig. 4). These findings suggest that FGF signaling enhances the initial integration of hiPS-Carts by the perichondrium-like membrane, but may inhibit the later stage of cartilage integration, thus reducing cartilage quality. To accelerate the healing of hiPS-Cart transplants, treatment with FGF18 should be limited to the initial stage.

We closely analyzed the integration of hiPS-Carts created from the QHJI hiPSC line. QHJI hiPSCs were generated from blood cells from a healthy individual homozygous for the most common HLA type in Japan. QHJI hiPSCs were generated at clinical grade for the purpose of using in clinical settings. Accordingly, we are planning to transplant hiPS-Carts generated from QHJI hiPSCs into articular cartilage defects of patients in an allogeneic manner in the near future. This study has clarified the integrating activity of hiPS-Carts in vitro and provides insight into the mechanism of the integration. We expect these findings to contribute to the understanding of the healing process of transplants and to the realization of regenerative treatment for articular cartilage damage.

\section{Acknowledgments}

We thank Shunsuke Kihara for the time-lapse imaging analysis, Takuya Yamamoto for the RNA sequencing analysis, Knut Woltjen for provision of AAVS1 reporter iPSC lines, and Peter Karagiannis for reading the article. This study was supported in part by Scientific Research Grants no. 15H02561 (to N.T.), no. 18H02923 (to N.T.), and 18H02924
Table 6. Number of QHJI Human Induced

Pluripotent Stem-Cart Pairs by Grade OF INTEGRATION IN RESPONSE TO NVP-BGJ398

\begin{tabular}{|c|c|c|c|c|}
\hline & Grade 0 & Grade 1 & $C \mathrm{cod}$ ? & \\
\hline & $\begin{array}{c}\text { No } \\
\text { integration }\end{array}$ & $\begin{array}{l}\text { Integration by } \\
\text { perichondrium- } \\
\text { like membrane }\end{array}$ & $\begin{array}{l}\text { Integration } \\
\text { by cartilage }\end{array}$ & Total \\
\hline \multicolumn{5}{|l|}{ Day 14} \\
\hline Vehicle & 4 & 5 & 6 & 15 \\
\hline $\begin{array}{l}\text { NVP- } \\
\text { BGJ398 }\end{array}$ & 15 & 0 & 0 & 15 \\
\hline
\end{tabular}

${ }^{\text {a }} P<0.01$ compared with vehicle by the Steel-Dwass test.

(to A.Y.) from MEXT, and the Practical Research Project for Rare/Intractable Diseases (step 0) Grants no. 17ek0109215h 0001 (to N.T.), Centers for Clinical Application Research on Specific Disease/Organ (type B) Grants no. 17bm0304004h 0005 (to N.T.), Core Center for iPS Cell Research Grants no. 17bm0104001h0005 (to N.T.), and the Acceleration Program for Intractable Diseases Research Utilizing Disease-Specific iPS Cells Grants no. 17bm0804006h0001 (to N.T.) from AMED.

\section{Disclosure Statement}

No competing financial interests exist.

\section{References}

1. Ma, B., Leijten, J.C., Wu, L., et al. Gene expression profiling of dedifferentiated human articular chondrocytes in monolayer culture. Osteoarthritis Cartilage 21, 599, 2013.

2. von der Mark, K., Gauss, V., von der Mark, H., and Müller, P. Relationship between cell shape and type of collagen synthesised as chondrocytes lose their cartilage phenotype in culture. Nature 267, 531, 1977.

3. Benya, P.D., Padilla, S.R., and Nimni, M.E. Independent regulation of collagen types by chondrocytes during the loss of differentiated function in culture. Cell 15, 1313, 1978.

4. Nixon, A.J., Begum, L., Mohammed, H.O., Huibregtse, B., O'Callaghan, M.M., and Matthews, G.L. Autologous chondrocyte implantation drives early chondrogenesis and organized repair in extensive full- and partial-thickness cartilage defects in an equine model. J Orthop Res 29, 1121, 2011.

5. Ansboro, S., Roelofs, A.J., and De Bari, C. Mesenchymal stem cells for the management of rheumatoid arthritis: immune modulation, repair or both? Curr Opin Rheumatol 29, 201, 2017.

6. Caplan, A.I. Adult mesenchymal stem cells: when, where, and how. Stem Cells Int 2015, 628767, 2015.

7. Caplan, A.I. MSCs: The sentinel and safe-guards of injury. J Cell Physiol 231, 1413, 2016.

8. Chesterman, P.J., and Smith, A.U. Homotransplantation of articular cartilage and isolated chondrocytes. An experimental study in rabbits. J Bone Joint Surg Br 50, 184, 1968.

9. Adkisson, H.D., Milliman, C., Zhang, X., Mauch, K., Maziarz, R.T., and Streeter, P.R. Immune evasion by neocartilagederived chondrocytes: implications for biologic repair of joint articular cartilage. Stem Cell Res 4, 57, 2010.

10. Erdmann, J. ISTO Technologies aims to rescue damaged joints. Chem Biol 18, 275, 2011. 
11. Farr, J., Tabet, S.K., Margerrison, E., and Cole, B.J. Clinical, radiographic, and histological outcomes after cartilage repair with particulated juvenile articular cartilage: A 2year prospective study. Am J Sports Med 42, 1417, 2014.

12. Tompkins, M., Adkisson, H.D., Bonner, K.F. DeNovo NT allograft. Oper Tech Sports Med 21, 82, 2013.

13. Adkisson, H.D.T., Martin, J.A., Amendola, R.L., et al. The potential of human allogeneic juvenile chondrocytes for restoration of articular cartilage. Am J Sports Med 38, 1324, 2010.

14. Huey, D.J., Hu, J.C., and Athanasiou, K.A. Unlike bone, cartilage regeneration remains elusive. Science 338, 917, 2012.

15. Takahashi, K., and Yamanaka, S. Induction of pluripotent stem cells from mouse embryonic and adult fibroblast cultures by defined factors. Cell 126, 663, 2006.

16. Takahashi, K., Tanabe, K., Ohnuki, M., et al. Induction of pluripotent stem cells from adult human fibroblasts by defined factors. Cell 131, 861, 2007.

17. Yamashita, A., Morioka, M., Yahara, Y., et al. Generation of scaffoldless hyaline cartilaginous tissue from human iPSCs. Stem Cell Rep 4, 404, 2015.

18. Yamashita, A., Morioka, M., Kishi, H., et al. Statin treatment rescues FGFR3 skeletal dysplasia phenotypes. Nature 513, 507, 2014.

19. Kimura, T., Yamashita, A., Ozono, K., and Tsumaki, N. Limited immunogenicity of human induced pluripotent stem cell-derived cartilages. Tissue Eng A 22, 1367, 2016.

20. Okita, K., Matsumura, Y., Sato, Y., et al. A more efficient method to generate integration-free human iPS cells. Nat Methods 8, 409, 2011.

21. Oceguera-Yanez, F., Kim, S.I., Matsumoto, T., et al. Engineering the AAVS1 locus for consistent and scalable transgene expression in human iPSCs and their differentiated derivatives. Methods 101, 43, 2016.

22. Bandyopadhyay, A., Kubilus, J.K., Crochiere, M.L., Linsenmayer, T.F., and Tabin, C.J. Identification of unique molecular subdomains in the perichondrium and periosteum and their role in regulating gene expression in the underlying chondrocytes. Dev Biol 321, 162, 2008.

23. Krejci, P. The paradox of FGFR3 signaling in skeletal dysplasia: why chondrocytes growth arrest while other cells over proliferate. Mutat Res Rev Mutat Res 759, 40, 2014.

24. Naski, M.C., Colvin, J.S., Coffin, J.D., and Ornitz, D.M. Repression of hedgehog signaling and BMP4 expression in growth plate cartilage by fibroblast growth factor receptor 3. Development 125, 4977, 1998.

25. Correa, D., Somoza, R.A., Lin, P., et al. Sequential exposure to fibroblast growth factors (FGF) 2, 9 and 18 enhances hMSC chondrogenic differentiation. Osteoarthritis Cartilage 23, 443, 2015.

26. Reker, D., Kjelgaard-Petersen, C.F., Siebuhr, A.S., et al. Sprifermin (rhFGF18) modulates extracellular matrix turnover in cartilage explants ex vivo. J Transl Med 15, 250, 2017.

Address correspondence to: Noriyuki Tsumaki, MD, PhD Cell Induction and Regulation Field Department of Clinical Application Center for iPS Cell Research and Application Kyoto University 53 Kawahara-cho Shogoin Sakyo-ku Kyoto 606-8507 Japan

E-mail: ntsumaki@cira.kyoto-u.ac.jp

Received: May 22, 2018

Accepted: July 16, 2018

Online Publication Date: September 21, 2018 